\title{
Compactación por el tráfico de la maquinaria agrícola: su efecto sobre el esfuerzo cortante del suelo y el rendimiento del cultivo de maíz (Zea mayz L.)
}

\section{Soil compaction produced by traffic machinery: effect on soil cutting effort and maize yield (Zea mayz L.)}

\author{
Virginia Susana Martiren¹, Roberto Andrés Fonterosa ${ }^{2}$, Xavier Bolívar Lastra-Bravo ${ }^{3}$, Guido Fernando Botta ${ }^{1,2}$ \\ ${ }^{1}$ Universidad Nacional de Luján, Dpto. Tecnología. Cruce de rutas 5 y 7, Luján C.P. 6700, Provincia de Buenos Aires, Argentina. \\ ${ }^{2}$ Universidad de Buenos Aires Facultad de Agronomía. Av. San Martin 4453 C.P. 1417 DSE, Buenos Aires, Argentina. \\ ${ }^{3}$ Universidad Central del Ecuador. Facultad de Ciencias Agrícolas. Carrera de Turismo Ecológico. Jerónimo Leiton y Av. \\ La Gasca s/n. Ciudadela Universitaria. Quito. 170521. Ecuador.
}

\begin{abstract}
Resumen
Se evaluó la compactación del suelo y los efectos de los residuos de la cosecha del maíz (Zea mays L.) sobre la emergencia de las plántulas y los rendimientos en tres diferentes métodos de labranza. El estudio se realizó durante cuatro estaciones de crecimiento sobre un suelo Argiudol típico. Se midieron: índice de cono, materia seca de la raíz por planta, materia seca por planta, emergencia de plántulas, y rendimiento. Los resultados mostraron que bajo siembra directa hubo menores rendimientos (entre el 10,7 y el 15,2\%) en comparación con el cincel y arado de reja y vertedera. En un principio, la emergencia fue más lenta para los tratamientos de suelo labrado, pero no se encontraron diferencias entre los tratamientos 18 días después de la siembra. Los valores más altos de materia seca de raíz se observaron en los suelos labrados con arado de cincel y de reja y vertedera (42,3 y 46,1 g planta ${ }^{-1}$, respectivamente). Se concluyó que el rendimiento del maíz está directamente relacionado con la compactación del suelo, y que las sembradoras de bajo peso utilizadas en los tratamientos que remueven el suelo produjeron compactación subsuperficial.
\end{abstract}

Palabras clave: sembradoras, siembra directa, cobertura del suelo, índice de cono, arado de reja y vertedera, arado escarificador de cinceles.

\begin{abstract}
Soil compaction and the effects of crop residues of maize (Zea mays L.) on seedling emergence and yields in three different tillage methods were evaluated. The study was conducted during four growing seasons on a Typical Argiudoll soil. The variables measured were: Cone Index, the root dry matter per plant, dry matter per plant, seedling emergence, and yield. The results showed that lower yields in soil under direct sowing treatment (between 10.7 and 15.2\%) compared with the chisel plow and moldboard plow. Initially, the emergence was slower for tilled soil treatments, but no differences between treatments were found 18 days after planting. The highest values of root dry matter per plant were observed in soil worked by chisel plow and moldboard plow (42.3 and $46.1 \mathrm{~g} \mathrm{plant}^{-1}$, respectively). The main conclusions were: corn yield is directly related to soil compaction, and lightweight planters used in treatments that remove the soil produced subsoil compaction.
\end{abstract}

Keywords: planters, direct sowing, soil residue cover, cone index, moldboard plow, chisel plow. 


\section{Introducción}

En la pasada década se publicaron conocimientos relacionados con prácticas conservacionistas, en la explotación de los suelos agrícolas. Sin embargo, hoy día siguen dándose reportes contradictorios en relación con los efectos de labranzas reducidas y la siembra directa (SD), sobre la compactación de estos suelos. Algunos investigadores han reportado efectos no significativos de los sistemas de labranza sobre la compactación de los suelos, a través de sucesivos cultivos (Blevins et al., 1977; Hill, 1990); en cambio Edwards et al. (1992) encontraron menores incrementos de la compactación en suelos bajo no laboreo frente a los que recibieron labranza convencional.

Por otro lado, se han encontrado sobrecompactaciones de suelos conducidos en ausencia de labranza o con labranza reducida (Voorhees \& Lindstrom, 1983; Botta et al., 2002). Finalmente, significativos incrementos en las compactaciones, que restringieron el desarrollo de los cultivos, han sido informados, mayoritariamente en cultivos de escarda, p. ej. maíz (Voorhees \& Lindstrom, 1983; Botta et al., 2006; Flowers \& Lal, 1998) y soja (Glycine max L. Merr.) (Al-Adawi \& Reeder, 1996; Botta et al., 2004; Botta et al., 2006). Horn (1998) concluyó que cerca del $20 \%$ de la degradación de los suelos alrededor del mundo se debían a la compactación por el tráfico agrícola. Solo en Europa 33 millones de hectáreas de tierras arables se encuentran altamente degradados por este problema.

En Argentina, 19-20 millones de hectáreas se siembran bajo el método de SD continua (AAPRESID, 2013; USDA, 2013), y una cantidad similar bajo el método de labranza convencional. $\mathrm{Su}$ clima se caracteriza por periodos de lluvias muy intensas, especialmente cuando los cultivos de verano no han cubierto completamente el suelo o no se han sembrado todavía. Los suelos predominantes tienen alto contenido de arcilla (suelos pesados), y el uso de la labranza conservacionista en ellos reduce la erosión, por lo menos $50 \%$ en comparación con los suelos desnudos (Botta et al., 2009; Botta et al., 2010). La labranza conservacionista incluye cualquier método de labranza o siembra que permite que, al menos, el $30 \%$ del suelo permanezca cubierto con los residuos vegetales de cultivos anteriores después de la siembra (EP291.2, ASAE Standard, 1993).
El método de SD tiene, generalmente, menor intensidad de tráfico que los utilizados en los métodos de labranza convencional. Pero, los rendimientos tienden a disminuir después de varios años de SD continua (Botta et al., 2006; Botta et al., 2009). Esta disminución podría resultar de una combinación de aumento de los problemas de control de malezas, enfermedades de la raíz, la compactación del suelo y los residuos de cultivos abundantes en la superficie del suelo.

Botta et al. (2004); Håkansson \& Reeder (1994); y Jorajuria et al. (1997) afirman, en forma preocupante, que la compactación del suelo ha sido durante mucho tiempo la causante de reducciones en el crecimiento de raíces y en el rendimiento de cultivos. Tanto la soja como los cultivos perennes son particularmente susceptibles a este problema, también el oeste de la región pampeana.

Oskui \& Voorhees (1990) manifestaron preocupación por el bajo interés investigativo del problema de la compactación, especialmente la inducida por el tráfico con altas cargas por eje. Las tendencias de la agricultura actual muestran una clara inclinación hacia el incremento de la potencia y, fundamentalmente, al tamaño de los equipos y máquinas.

Flowers \& Lal (1998) no encuentran demasiada influencia de los tratamientos previos de labranza, sobre la distribución vertical de la compactación, en un suelo Ochraqualf mólico, fino, illítico, mésico. Sin embargo, sí encontraron respuesta para las diferentes intensidades de tráfico (Mg.km.ha-1) hechas sobre la base del pasaje con cargas de 10 y $20 \mathrm{Mg}$ por eje y el testigo sin tráfico. Taylor et al. (1966) midieron el número de raíces primarias de plantas de algodón que penetró en capas compactadas de los diferentes suelos en ambientes semiáridos en los EE.UU. Encontraron que el número de raíces que penetran el suelo disminuyó notablemente a medida que la resistencia a la penetración se acercó a $2 \mathrm{MPa}$.

Así también, Botta et al. (2013) indican que en suelos extremadamente densos o compactados se impide el crecimiento de las raíces y de este modo se limita el consumo de agua a la planta, lo que afecta su rendimiento. La respuesta de la raíz a la compactación puede ser compleja debido a las numerosas formas en las que ésta puede modificar las propiedades físicas del suelo. 
En esta situación de compactación, el maíz muestra una pobre capacidad de macollaje, lo que se traduce en una reducción de su capacidad para compensar una baja densidad de plantas (Tourn et al., 2003). Por otra parte, si las plantas no llegan al umbral de crecimiento adecuado durante el período comprendido entre quince días antes y quince días después de la floración, serán estériles.

En este marco surge como relevante la necesidad de conocer la reología del suelo bajo diferentes intensidades de tráfico, derivados de al menos dos sistemas de conducción: el laboreo convencional versus la SD. En este último caso, la presencia de un suelo con tapiz vegetal al momento del tráfico, ha mostrado evidencias suficientes de un comportamiento reológico diferente. Debido a esto es necesario su estudio a modo de establecer el verdadero impacto del tráfico sobre las propiedades físico-mecánicas, bajo una hipótesis básica de sustentabilidad del sistema.

Los objetivos del estudio fueron cuantificar el efecto de la compactación del suelo y los residuos de cosecha sobre la emergencia de plántulas y el rendimiento del cultivo de maíz (RM), en el partido de Luján provincia de Buenos Aires, durante cuatro estaciones de crecimiento, empleando tres métodos de labranza diferentes (siembra directa (SD), arado de cincel $(\mathrm{CN})$ y arado de reja y vertedera $(\mathrm{AV}))$.
Las hipótesis de trabajo de este estudio son las siguientes: (a) los rendimientos del maíz se ven afectados por el método de labranza utilizado para la preparación del suelo y (b) es posible diagnosticar el estado de compactación superficial de un suelo a partir de los datos de esfuerzo cortante y relacionarlo al rendimiento del cultivo de maíz.

\section{Materiales y métodos}

\subsection{El sitio}

El experimento se llevó a cabo en el este de la región Pampeana Argentina a los $34^{\circ} 32^{\prime}$ $30 \mathrm{~S}, 59^{\circ} 07^{\prime} 50 \mathrm{O}$ en el partido de Luján. La altitud del sitio es de $22 \mathrm{~m}$ s.n.m. La pendiente es de tipo 1 , con un gradiente de $0,5 \%$. El suelo es un Argiudol típico (Soil Conservation Service, 1994), con un contenido de materia orgánica que va desde el 3,6\% (w/w) en la superficie hasta el $1,4 \%$ a una profundidad de $0,6 \mathrm{~m}$. La historia de manejo del suelo incluye 10 años de rotación de cultivos, siguiendo un patrón muy común en la región, el trigo de invierno, seguido por el maíz en el verano. El perfil típico del suelo se puede ver en la Tabla 1.

Tabla 1. Perfil del suelo

\begin{tabular}{|c|c|c|c|c|c|c|c|c|}
\hline HORIZONTES & Ap & A12 & $\mathrm{B} 1$ & $\mathrm{~B} 21 \mathrm{t}$ & $\mathrm{B} 22 \mathrm{t}$ & $\mathrm{B} 23 \mathrm{t}$ & B3 & Cca \\
\hline Profundidad $(\mathrm{cm})$ & $0-15$ & $16-22$ & $23-35$ & $35-60$ & $65-80$ & $90-110$ & $120-150$ & $160-220$ \\
\hline Carbono Orgánico $\left(\mathrm{g} \mathrm{kg}^{-1}\right)$ & 17.4 & 13.5 & 9.3 & 6.3 & 5.0 & 3.1 & 2.2 & 1.4 \\
\hline Nitrógeno Total ( $\mathrm{g} \mathrm{kg}^{-1}$ ) & 2.4 & 1.4 & 1.0 & 0.85 & 0.75 & 0.58 & 0.42 & - \\
\hline Arcilla $(<2 \mathrm{~m})\left(\mathrm{g} \mathrm{kg}^{-1}\right)$ & 212 & 255 & 249 & 332 & 475 & 330 & 230 & 158 \\
\hline $\operatorname{Limo}(2-20 \mathrm{~m})\left(\mathrm{g} \mathrm{kg}^{-1}\right)$ & 32.3 & 34.7 & 29.8 & 29.3 & 20.8 & 31.2 & 32.9 & 287 \\
\hline $\operatorname{Limo}(2-50 \mathrm{~m})\left(\mathrm{g} \mathrm{kg}^{-1}\right)$ & 756 & 708 & 672 & 613 & 500 & 630 & 727 & 799 \\
\hline Arena Fina $(100-250 \mathrm{~m})\left(\mathrm{g} \mathrm{kg}^{-1}\right)$ & 3.0 & 2.0 & 3.0 & 4.0 & 4.0 & 4.0 & 5.0 & 4.0 \\
\hline Arena gruesa $(500-1000 \mathrm{~m})\left(\mathrm{g} \mathrm{kg}^{-1}\right)$ & 0.0 & 0.0 & 0.0 & 0.0 & 0.0 & 0.0 & 0.0 & 0.0 \\
\hline $\mathrm{pH}$ in $\mathrm{H}_{2} 0(1: 2.5)$ & 5.8 & 5.8 & 6.0 & 6.2 & 6.5 & 6.4 & 6.4 & 7.9 \\
\hline \multicolumn{9}{|l|}{ Cationes de cambio (m.e. $100 \mathrm{~g}^{-1}$ ) } \\
\hline $\mathrm{Ca}^{2+}$ & 11.4 & 12.7 & 12.0 & 13.8 & 18.3 & 17.2 & 16.5 & - \\
\hline $\mathrm{Mg}^{2+}$ & 2.9 & 2.5 & 3.1 & 4.5 & 6.5 & 6.4 & 3.8 & - \\
\hline $\mathrm{Na}^{+}$ & 0.2 & 0.1 & 0.2 & 0.1 & 0.2 & 0.2 & 0.3 & 0.5 \\
\hline $\mathrm{K}^{+}$ & 1.4 & 1.0 & 0.9 & 1.3 & 2.3 & 2.4 & 2.3 & 2.4 \\
\hline
\end{tabular}

\subsection{Los tratamientos de labranza}

Se utilizaron tres métodos de labranza o tratamientos, cuyas principales características se muestran en la Tabla 2. Doce parcelas de $100 \mathrm{~m}$ x $7 \mathrm{~m}$ (con 10 filas a 0,70 m) fueron asignados aleatoriamente con cuatro repeticiones para cada tratamiento de labranza separadas a 20 metros una 
de otra como zonas de amortiguamiento o buffer y, así, evitar interacciones (Figura 1). La siembra y la cosecha se realizaron en las fechas comunes a la zona del ensayo.

Tabla 2. Parcelas de ensayo, en el centro de la imagen, parcela bajo siembra directa.

\begin{tabular}{|clccc|}
\hline $\begin{array}{c}\text { Tratamientos de } \\
\text { labranza }\end{array}$ & Descripción y secuencia & $\begin{array}{c}\text { Número de } \\
\text { pasadas/ hectárea }\end{array}$ & $\begin{array}{c}\text { Peso Total } \\
(\mathrm{kN})\end{array}$ & $\begin{array}{c}\text { Total Desplazamiento } \\
\left(\mathrm{km} \mathrm{ha} \mathrm{h}^{-1}\right)\end{array}$ \\
\hline $\mathrm{SD}$ & $\begin{array}{l}\text { Pulverizado (pre-siembra) } \\
\text { y siembra directa }\end{array}$ & 2 & 187 & 3.11 \\
\hline $\mathrm{CN}$ & $\begin{array}{l}\text { Cincel de 11 arcos curvos rígidos - } \\
\text { rastra de discos (600 N/disk, 30 disk } \\
\text { - Rastra de dientes de 8 secciones y } \\
\text { 2 pasadas de rolos destarronadores }\end{array}$ & 5 & 60.4 & 7.1 \\
\hline $\mathrm{AV}$ & $\begin{array}{l}\text { Arado de reja y vertedera - 2 pases } \\
\text { de rastra de discos liviana en } \\
\text { tandem con un cultivador de campo } \\
\text { y 1 pasada de rastra de dientes- }\end{array}$ & 4 & 94.4 & 10.98 \\
\hline
\end{tabular}

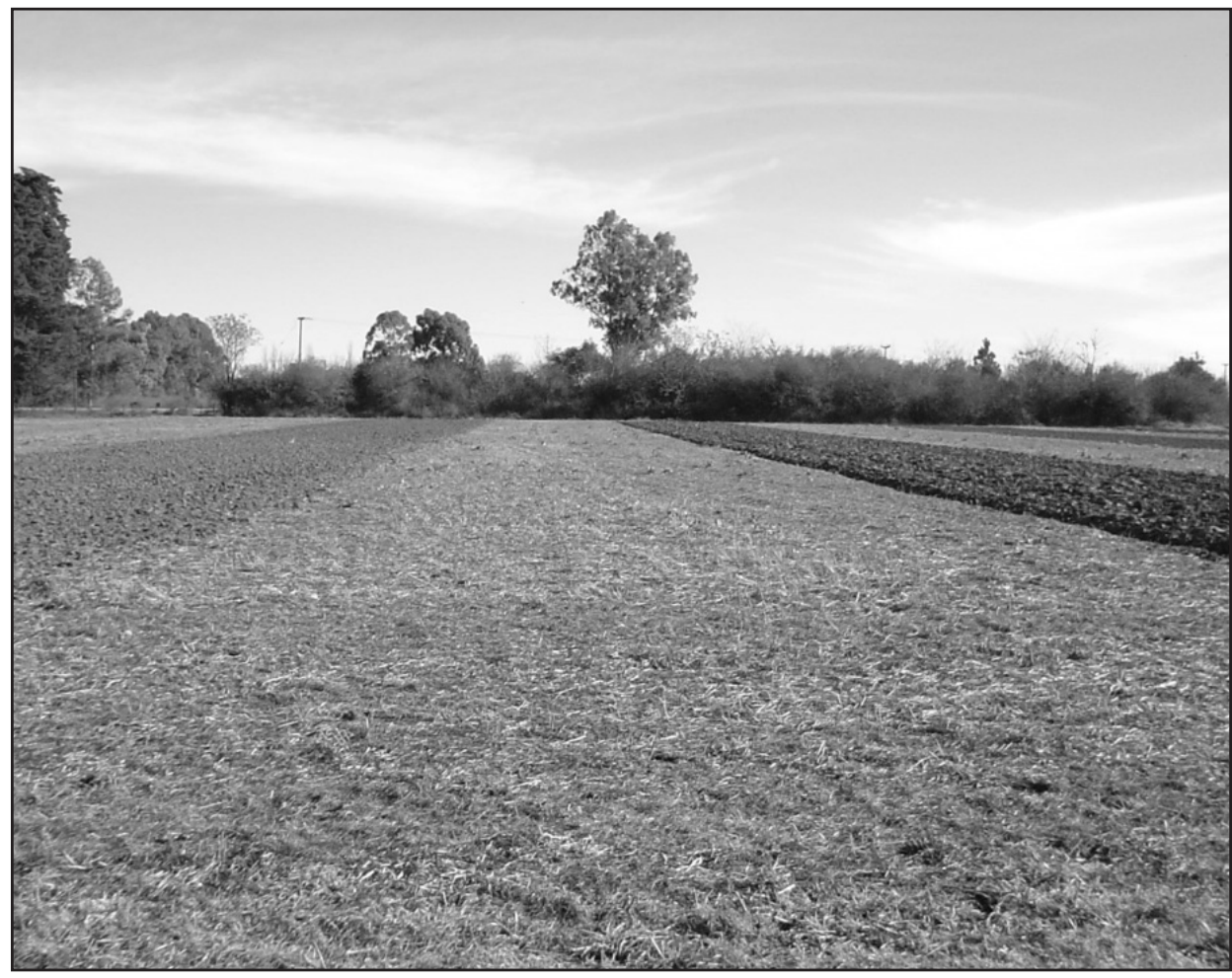

Figura 1. Parcelas de ensayo, en el centro de la imagen, parcela bajo siembra directa.

\subsection{Tractores y sembradoras}

Un tractor J. Deere $(160 \mathrm{CV} / 117 \mathrm{~kW}$ del motor, Figura 2) (TDA), cuyo peso es de 98 kN (39,2 y 58,8 $\mathrm{kN}$ en los ejes delantero y trasero, respectivamente), se utilizó a una velocidad de desplazamiento de 5,5 $\mathrm{km} \mathrm{h}^{-1}$. Neumáticos delanteros nuevos rodados 16.9 R 28 y traseros rodados 20.1 R 30 inflados a 100 $\mathrm{kPa}$ y $80 \mathrm{kPa}$ respectivamente, dentro de los límites aconsejados por Firestone Neumáticos Agrícolas. 


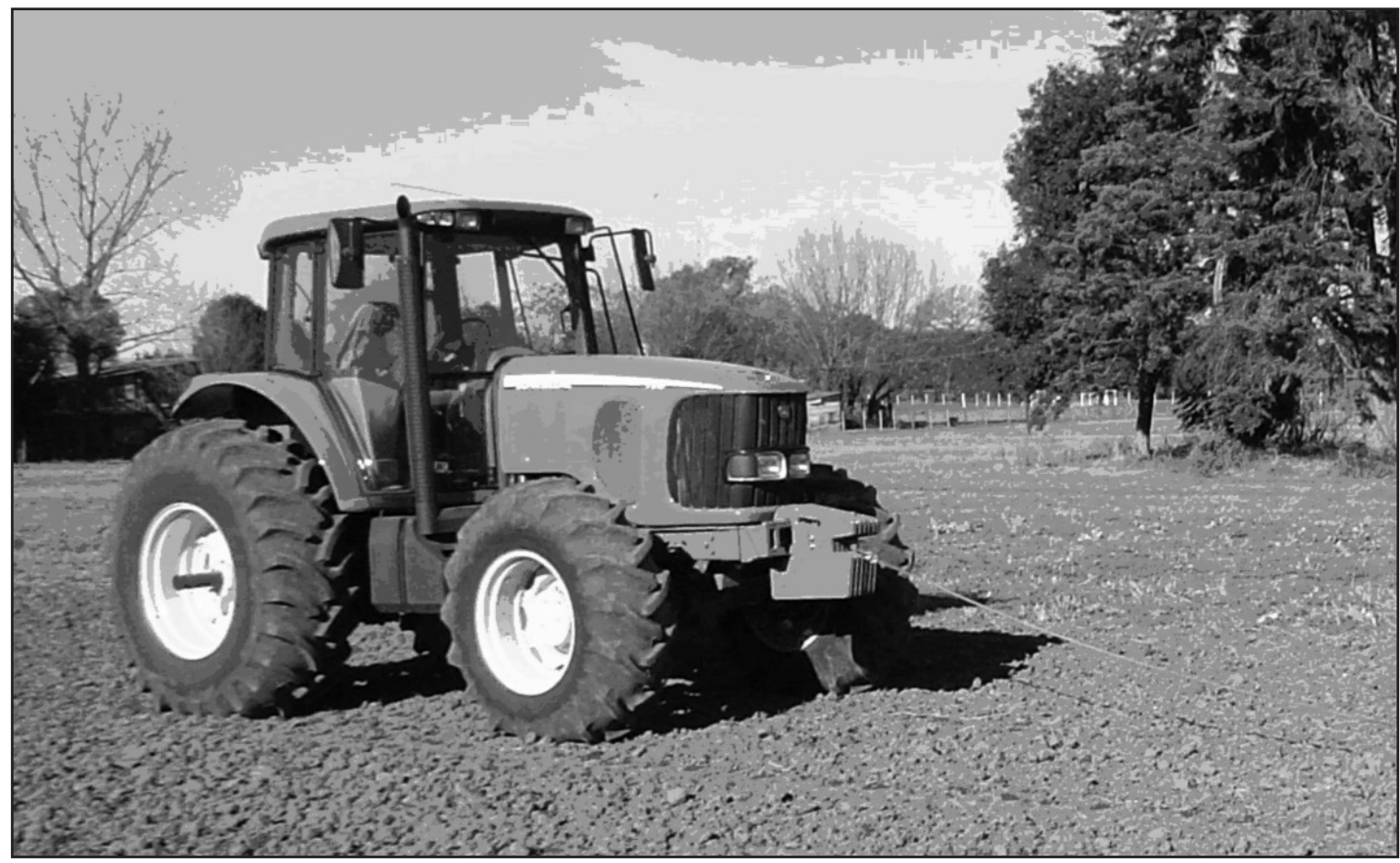

Figura 2. John Deere 7515, utilizado en todos los tratamientos del ensayo.

El área de contacto del neumático/suelo fue 0.400 y $0.873 \mathrm{~m}^{2}$, y la presión sobre el suelo fue de 49 y $33.7 \mathrm{kPa}$, en la parte delantera y trasera, respectivamente. Estas mediciones se llevaron a cabo en el campo experimental según método de Botta et al. (2004).
En el ensayo se utilizaron dos sembradoras diferentes (Tabla 3, Figuras 3 y 4). El tratamiento bajo SD fue traficado con una pasada del tractor J. Deere 7515 y la sembradora 1 , mientras que los tratamientos $\mathrm{CN}$ y AV, fueron traficados con una pasada del mismo tractor pero utilizando la sembradora 2 .

Tabla 3. Características de las sembradoras

\begin{tabular}{|lcc|}
\hline \multicolumn{1}{|c|}{ Especificaciones } & $\begin{array}{c}\text { Super Walter } \\
\text { Sembradora 1 }\end{array}$ & $\begin{array}{c}\text { John Deere 1700 Max Emerge } \\
\text { Sembradora 2 }\end{array}$ \\
\hline & & \\
Peso total cargada $(\mathrm{kN})$ & 69.6 & 14.9 \\
Ancho $(\mathrm{mm})$ & 7000 & 5600 \\
Número de unidades de siembra & 10 & 8 \\
Distancia entre filas $(\mathrm{mm})$ & 700 & 700 \\
Sistema dosificador & Plato semillero & Neumático \\
Neumáticos & $400 / 60-15.5$ & $7.60-15$ \\
Presión de Contacto $(\mathrm{kPa})$ & 122 & 91.6 \\
Tren de siembra & Cuchilla turbo +2 discos & Doble discos con 2 ruedas \\
& abresurcos & limitadoras de profundidad \\
& & \\
\hline
\end{tabular}

Ambos, el tractor y las dos sembradoras, son utilizados comúnmente por los productores del área de estudio. La sembradora 1 se utilizó en el tratamiento $\mathrm{SD}$, y la sembradora 2 en $\mathrm{CN}$ y $\mathrm{AV}$, de acuerdo con sus características (Fonterosa, 2012). 


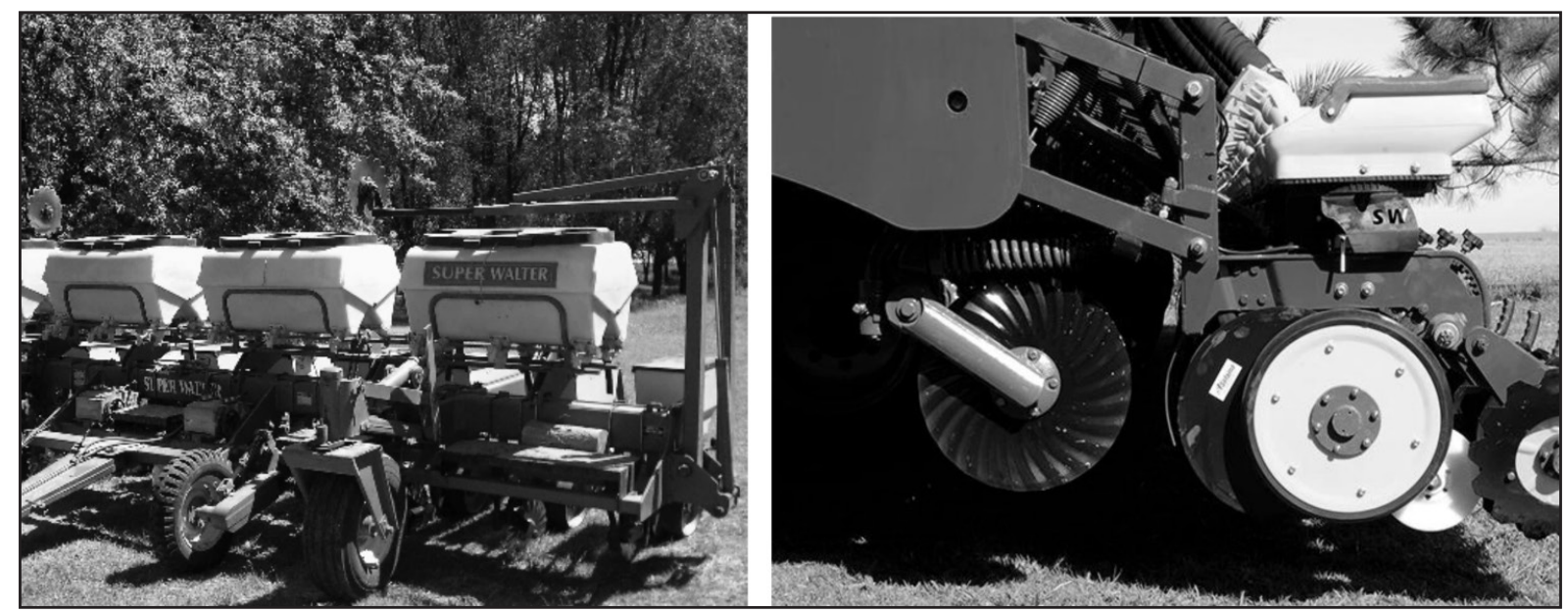

Figura 3. Sembradora 1, Super Walter y detalle del tren de siembra.
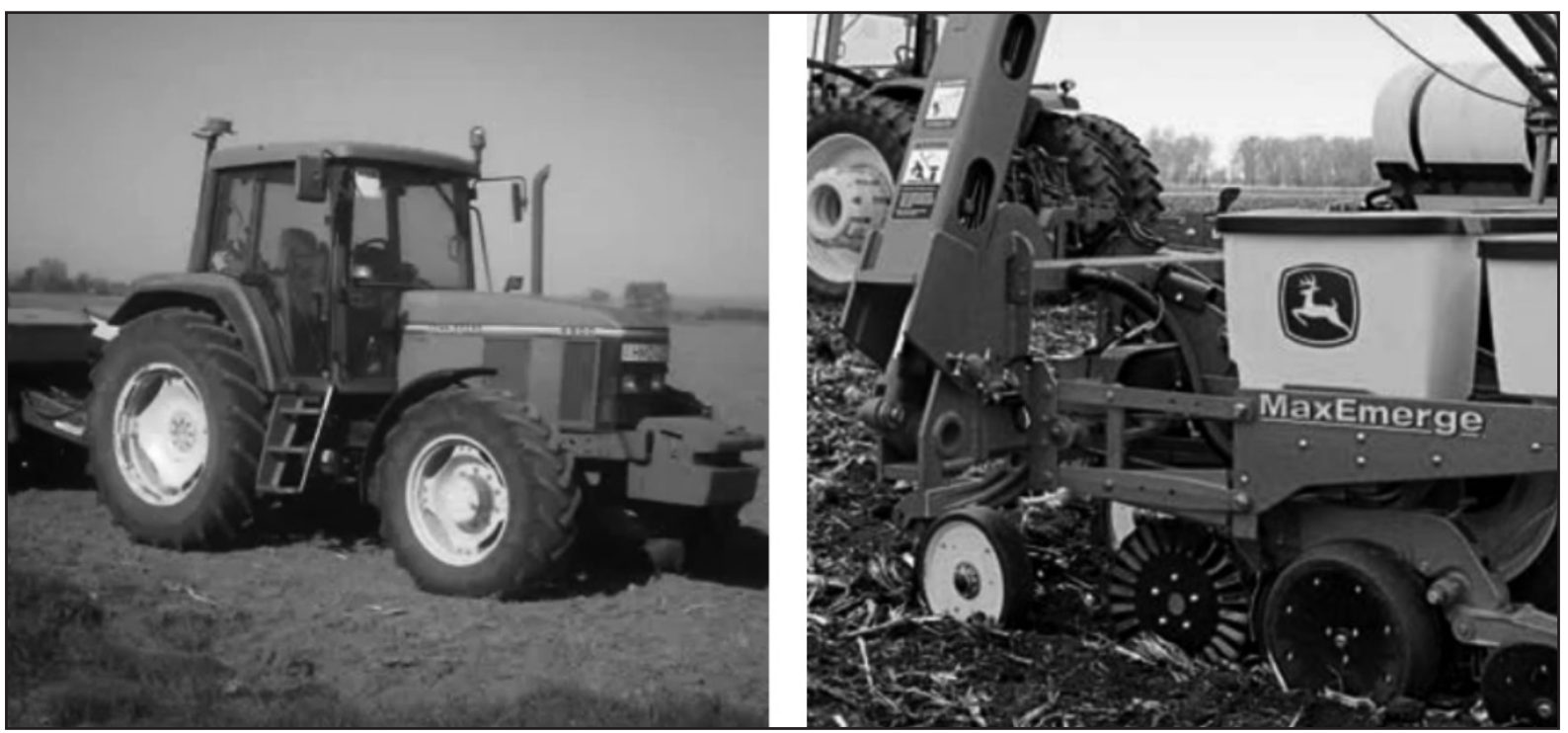

Figura 4. Tractor y sembradora 2, J. Deere 1700 Maxemerge, y detalle del tren de siembra.

\subsection{Variables experimentales medidas}

El número de plantas emergidas por metro lineal se observó en todas las filas y huellas del tractor. La muestra consistió en 320 observaciones en cada caso (huella y fila) (40 observaciones / fila x 8 líneas / réplica). Los conteos fueron realizados 11,14 y 18 días después de la siembra.

Antes de la siembra (pre-siembra), se determinó la cobertura de rastrojos por el método de la transecta. El contenido de agua del suelo (w/w) y el índice de cono (IC) se midieron el mismo día des- pués de la siembra. Las fechas de siembra fueron las comunes para la zona de estudio.

El w/w se midió con una sonda Troxler 3440 de rayos gamma en una profundidad que oscila de 0 a $150 \mathrm{~mm}$ y $150-300 \mathrm{~mm}$ y en una trinchera de 300 a $450 \mathrm{~mm}$ de profundidad. En los suelos con alto contenido de materia orgánica, como ocurrió en este caso, la sonda de neutrones puede subestimar el w/w considerablemente. Por consiguiente, este parámetro se verificó mediante el método de cilindro (Figura 5). 


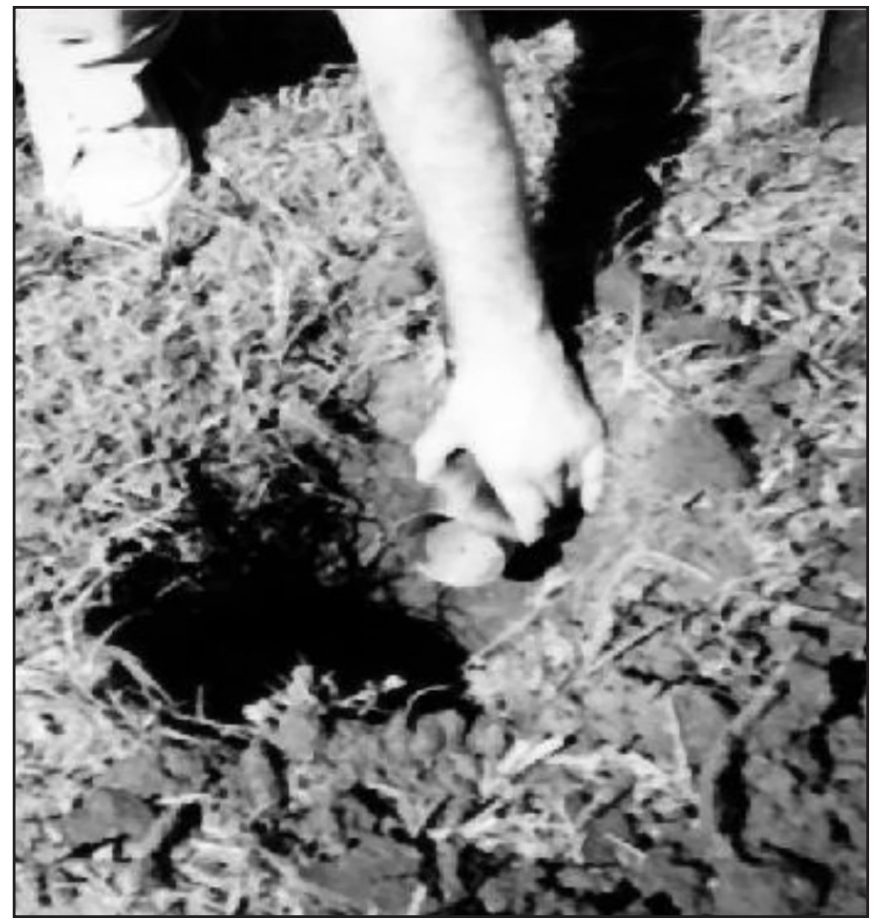

Figura 5. Cilindro para la medición y chequeo del contenido de agua en el suelo.

El índice de cono (IC) se determinó con un penetrómetro Scout 900 (Figura 6) con grabación de datos. El cono cumple con las medidas según el estándar S 313 de
ASAE Standards (1992). Cada dato representa el promedio de 25 muestras tomadas de cada parcela en el 0 - a rango de profundidad de $450 \mathrm{~mm}$, a intervalos de $25 \mathrm{~mm}$.

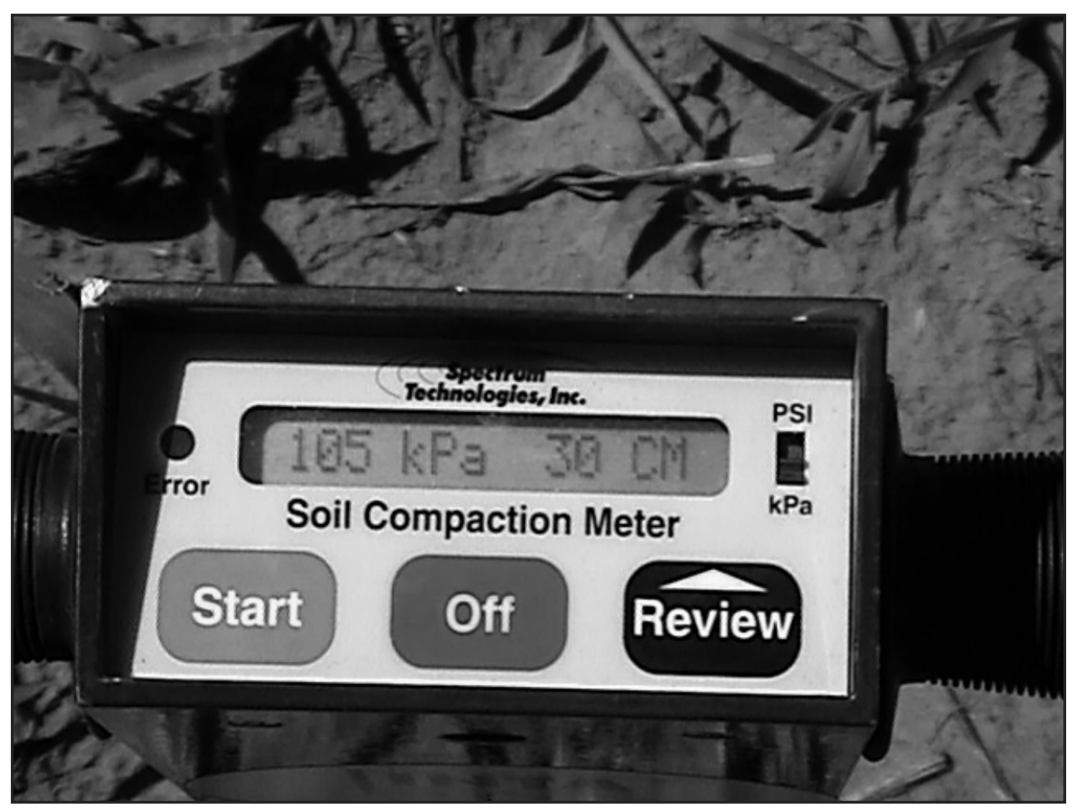

Figura 6. Detalle del display del penetrómetro. 
El w/w e IC se midieron en lugares al azar en todas las parcelas. Los datos recogidos para el IC antes de la siembra (parcela control), proporcionaron una clara indicación de la condición inicial del suelo en cada tratamiento de labranza. Los efectos de los tratamientos de tráfico en el suelo se midieron en el momento de la siembra en las líneas centrales de la huellas de los neumáticos debido a que los efectos de compresión tienden a concentrarse en este punto (Söhne, 1958).

La materia seca de la raíz (MSR) y la materia seca por planta (MSP) se midieron 8 semanas después de la emergencia del cultivo (en floración). Se tomaron muestras de raíces en el 0 - a rango de profundidad de $300 \mathrm{~mm}$. La MSR se midió a $300 \mathrm{~mm}$ de profundidad sólo porque la mayoría de las raíces de este cultivo se concentran en los primeros $250 \mathrm{~mm}$. Un total de 70 muestras fueron tomadas por trata- miento. Para determinar el peso seco, las raíces, primero se lavaron y luego se secaron a $104{ }^{\circ} \mathrm{C}$ en un horno convencional hasta peso constante

Para la determinación de MSP, las plantas se cortaron justo por encima del nivel del suelo, luego fueron lavados en agua destilada y se secaron en estufa a $104{ }^{\circ} \mathrm{C}$ hasta peso constante.

El esfuerzo cortante, para cada condición de suelo, se midió con una placa de corte de $0.48 \mathrm{~m}$ de largo x $0.18 \mathrm{~m}$ de ancho, y dispone de 4 garras de $25 \mathrm{~mm}$ de altura (Figura 7), ubicadas en ángulo de $90^{\circ}$ respecto a la dirección de avance (Botta, 2000). El esfuerzo cortante resultante de los ensayos se midió con un dinamómetro de funcionamiento hidráulico, y se realizaron lecturas por medio de un manómetro para determinar el esfuerzo requerido para cortar el suelo en cada uno de los tratamientos.

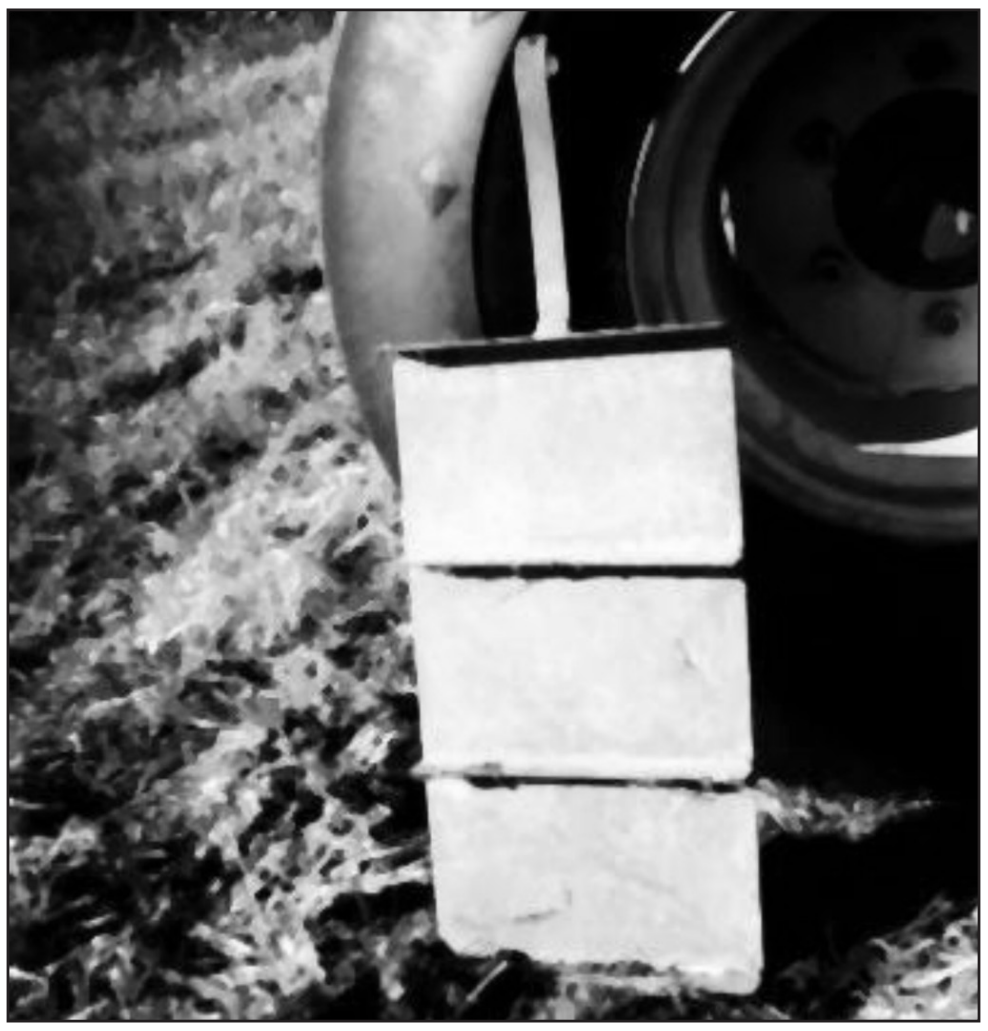

Figura 7. Detalle de la placa de corte.

Todos los datos fueron sometidos a un análisis de varianza (ANOVA), y las medias fueron analizadas por la prueba de rangos múltiples de Duncan utilizando el programa Statgraf 7.1 (Botta et al., 2010).
Para analizar los efectos de la compactación del suelo y la cubierta de residuos (factores) y su interacción en la emergencia de las plántulas de maíz, se utilizó un procedimiento de ANOVA de dos factores. 


\section{Resultados y discusión}

3.1. Contenido de agua del suelo, condiciones climáticas y las condiciones iniciales del suelo del ensayo

El w/w, promedio para los cuatro años de ensayo en el día de siembra fue del $18.6 \%$ del peso seco desde los 0 hasta los $150 \mathrm{~mm}$ de profundidad, $20 \%$ de 150 a $300 \mathrm{~mm}$ y $20.7 \%$ desde 300 hasta $450 \mathrm{~mm}$. La precipitación total cada diez días y el promedio de temperaturas máximas del 1/10 al 31/03 para cada año se muestra en la Figura 8.

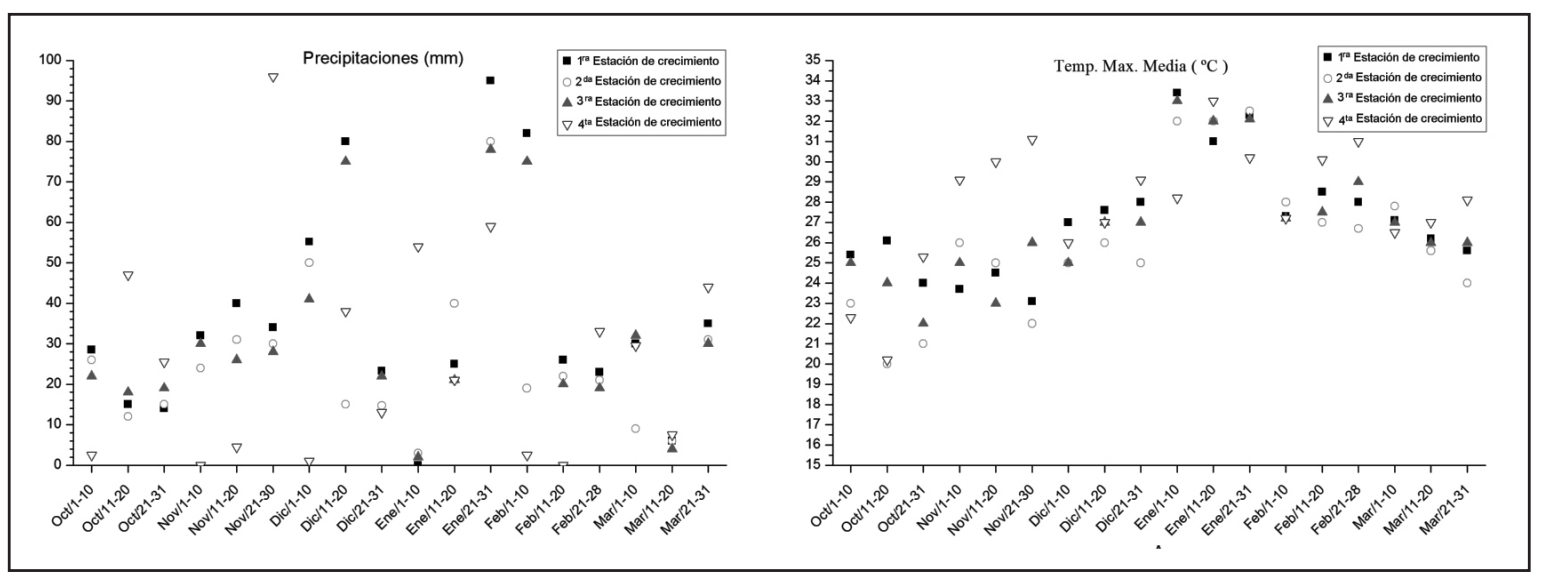

Figura 8. Precipitaciones totales y promedio de la temperatura máxima.

La temperatura máxima media del aire fue moderada en octubre, noviembre, diciembre, febrero y marzo, pero superó los $32^{\circ} \mathrm{C}$ en enero. La precipitación en octubre fue adecuada para la emergencia de las plántulas. Debido a que la lluvia fue abundante antes de la cosecha (durante los últimos 10 días del mes de marzo), el w/w fue alto. De acuerdo con nuestro estudio del ciclo completo del cultivo, la temperatura fue normal para el verano, dando plantas de importante tamaño (Figura 9). De acuerdo con Tourn et al. (2003), la temperatura afecta a la longitud del ciclo de cultivo desde la siembra hasta que alcanza la madurez fisiológica, mientras que el fotoperiodo puede afectar el tiempo entre la brotación y la floración.

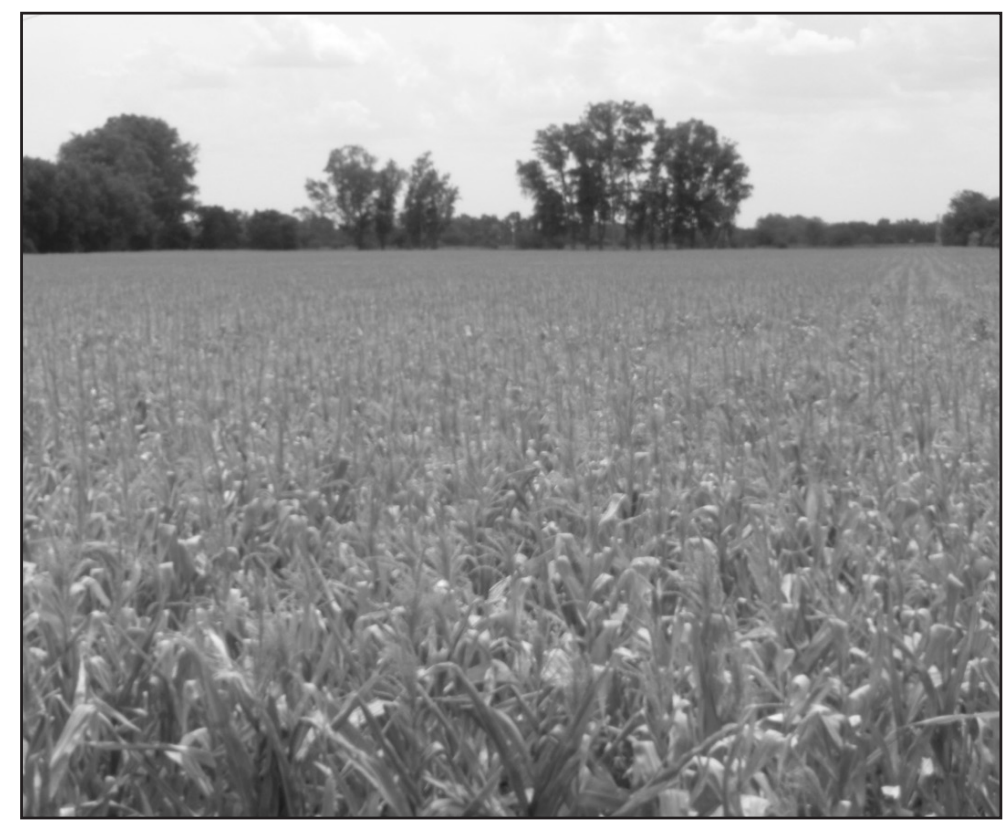

Figura 9. Sitio del ensayo de los tres tratamientos de labranza. 
Durante la temporada de producción, las condiciones meteorológicas fueron muy similares en los cuatro años de estudio. Por lo tanto, cualquier variación en los RM sería el resultado de los dife- rentes tratamientos de labranza del suelo. La Figura 10 muestra el IC antes de la siembra (pre-siembra) y ofrece una clara indicación de la condición inicial del suelo en cada tratamiento.

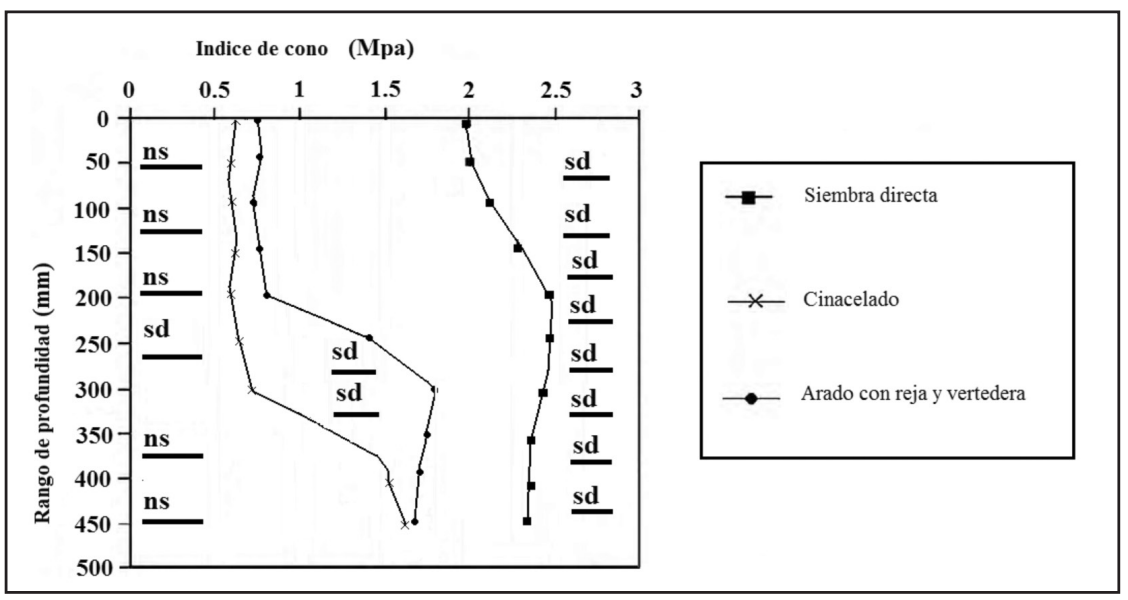

Figura 10. Valores de IC (pre-siembra).

Se observa que el IC en el tratamiento SD fue superior a $2 \mathrm{MPa}$ en el rango de profundidad de 0 a $450 \mathrm{~mm}$, y que los efectos fueron muy diferentes para AV y CN. El IC de AV y CN fue estadísticamente diferente $(\mathrm{p}<0,01)$ que el de $\mathrm{SD}$, pero a distintas profundidades (como era de esperar), $280 \mathrm{~mm}$ para CN y $200 \mathrm{~mm}$ para AV.

El análisis de varianza de los valores de IC promedio en las profundidades de 200 a $350 \mathrm{~mm}$ mostró una diferencia significativa entre $\mathrm{CN}$ y $\mathrm{AV}$, siendo mayor para este último.

\subsection{Residuos vegetales y la compactación del suelo}

Los efectos de los residuos vegetales luego de la cosecha sobre la compactación del suelo y su interacción sobre la emergencia de las plántulas fueron analizados. El porcentaje de residuo o rastrojo remanente sobre el suelo después de la labranza fue significativamente mayor en la SD (fuera del surco de siembra) y en el CN que en el AV. Botta et al. (2009) encontraron que el uso continuado de SD (año tras año) resultó en una acumulación de residuos de cultivos y compactación del suelo (Tabla 4).

Tabla 4. Efecto de la compactación del suelo (expresada como Índice de cono 0 - $150 \mathrm{~mm}$ ) y el rastrojo sobre la emergencia del maíz.

\begin{tabular}{|c|c|c|c|c|c|c|c|c|c|c|c|c|c|c|}
\hline \multirow{4}{*}{$\begin{array}{c} \\
\begin{array}{c}\text { Tratamientos } \\
\text { de labranza }\end{array}\end{array}$} & \multirow{4}{*}{$\begin{array}{c}\mathrm{IC} \\
(\mathrm{kPa})\end{array}$} & \multirow{4}{*}{$\begin{array}{l}\text { Rastrojo } \\
\text { en sup. } \\
(\%)\end{array}$} & \multicolumn{3}{|c|}{$\begin{array}{c}\mathbf{1}^{\mathbf{0}} \text { Estación de } \\
\text { crecimiento } \\
2004-2005 \\
\end{array}$} & \multicolumn{3}{|c|}{$\begin{array}{c}2^{\circ} \text { Estación de } \\
\text { crecimiento } \\
2005-2006 \\
\end{array}$} & \multicolumn{3}{|c|}{$\begin{array}{c}3^{\circ} \text { Estación de } \\
\text { crecimiento } \\
2006-2007 \\
\end{array}$} & \multicolumn{3}{|c|}{$\begin{array}{c}4^{0} \text { Estación de } \\
\text { crecimiento } \\
2007-2008 \\
\end{array}$} \\
\hline & & & \multicolumn{12}{|c|}{ Emergencia del maíz } \\
\hline & & & \multicolumn{3}{|c|}{$\begin{array}{l}\text { Días desde la } \\
\text { siembra }\end{array}$} & \multicolumn{3}{|c|}{$\begin{array}{c}\text { Días desde la } \\
\text { siembra }\end{array}$} & \multicolumn{3}{|c|}{$\begin{array}{l}\text { Días desde la } \\
\text { siembra }\end{array}$} & \multicolumn{3}{|c|}{$\begin{array}{c}\text { Días desde la } \\
\text { siembra }\end{array}$} \\
\hline & & & 11 & 14 & 18 & 11 & 14 & 18 & 11 & 14 & 18 & 11 & 14 & 18 \\
\hline AV & $1.13 \mathrm{a}$ & $12.1 \mathrm{a}$ & $0.35 \mathrm{a}$ & $2.80 \mathrm{a}$ & $4.50 \mathrm{a}$ & $0.30 \mathrm{a}$ & $2.60 \mathrm{a}$ & $5.00 \mathrm{a}$ & $0.35 \mathrm{a}$ & $2.20 \mathrm{a}$ & $5.50 \mathrm{a}$ & $0.41 \mathrm{a}$ & $2.28 \mathrm{a}$ & $5.7 \mathrm{a}$ \\
\hline $\mathrm{CN}$ & $1.17 \mathrm{a}$ & $40.1 \mathrm{~b}$ & $0.25 \mathrm{a}$ & $2.71 \mathrm{a}$ & $5.00 \mathrm{a}$ & $0.35 \mathrm{a}$ & $2.30 \mathrm{a}$ & $4.80 \mathrm{a}$ & $0.31 \mathrm{a}$ & $2.80 \mathrm{a}$ & $5.00 \mathrm{a}$ & $0.38 \mathrm{a}$ & $3.01 \mathrm{a}$ & $5.2 \mathrm{a}$ \\
\hline $\begin{array}{l}\text { SD (en el } \\
\text { surco) }\end{array}$ & $2.62 \mathrm{~b}$ & $11.4 \mathrm{a}$ & $1.50 \mathrm{~b}$ & $4.50 \mathrm{~b}$ & $5.70 \mathrm{a}$ & $1.60 \mathrm{~b}$ & $5.10 \mathrm{~b}$ & $5.80 \mathrm{a}$ & $1.61 \mathrm{~b}$ & $5.20 \mathrm{~b}$ & $5.70 \mathrm{a}$ & $1.60 \mathrm{a}$ & $5.2 \mathrm{~b}$ & $5.8 \mathrm{a}$ \\
\hline
\end{tabular}


Una investigación realizada, sobre las prácticas de siembra en la zona de estudio, reveló que los residuos de las plantas de maíz son un obstáculo importante para la siembra de los cultivos posteriores en SD, sobre todo con sembradoras que no utilizan discos abresurcos. En el cultivo de maíz, la limpieza del rastrojo en superficie ayuda a la siembra y también acelera el calentamiento del suelo.

En los suelos trabajados bajo SD, la cobertura de residuos vegetales en el surco depende de la utilización del sistema abresurco de la sembradora. Los sistemas abresurcos de cuchilla turbo y un abridor de doble disco son los mas difundidos. La menor cantidad de residuo que queda en el surco fue principalmente el resultado de la gran cantidad de tierra removida por la cuchilla turbo (Tabla 4).

De acuerdo con Tourn et al. (2003), la remoción del rastrojo por acción de la cuchilla turbo mejoró significativamente la emergencia de plántulas en el tratamiento bajo SD respecto a los convencionales (CN y AV). Sin embargo, no hubo diferencias en la emergencia de plántulas (Tabla 4).

El estudio mostró que la compactación causada por la maquinaria pesada (tractor y sembradora 1) produjo grandes cambios en las propiedades físicas del suelo a nivel superficial y del subsuelo respecto a lo que produjo el equipo liviano (sembradora 2 y tractor). Estos resultados son consistentes con los de Botta et al. (2006), que mostraron que los efectos de la compactación producida por una alta carga por eje se relaciona con el tipo de suelo, el número de pasadas y el número de años transcurridos desde el inicio de la compactación. En la primera estación de crecimiento, el aumento del IC, como resultado del tráfico de siembra, fue particularmente marcado en intervalo de 0 a 300 $\mathrm{mm}$ de profundidad, aunque el aumento de la resistencia a la penetración en los tratamientos de $\mathrm{CN}$ y $\mathrm{AV}$ fueron menores que en SD (Tabla 5).

Tabla 5. Valores medios de IC (MPa) después de la siembra

\begin{tabular}{|c|c|c|c|c|c|c|c|c|c|c|c|c|}
\hline & \multicolumn{12}{|c|}{ Estaciones de Crecimiento } \\
\hline & \multicolumn{3}{|c|}{ Siembra 2004} & \multicolumn{3}{|c|}{ Siembra 2005} & \multicolumn{3}{|c|}{ Siembra 2006} & \multicolumn{3}{|c|}{ Siembra 2007} \\
\hline & \multicolumn{12}{|c|}{ Tratamientos } \\
\hline Nivel de Prof. (mm) & SD & $\mathrm{CN}$ & AV & $\mathrm{SD}$ & $\mathrm{CN}$ & AV & SD & $\mathrm{CN}$ & AV & SD & $\mathrm{CN}$ & $\mathrm{AV}$ \\
\hline 0 & $2.50 \mathrm{~b}$ & $1.10 \mathrm{a}$ & $0.97 \mathrm{a}$ & $2.61 \mathrm{~b}$ & $0.9 \mathrm{a}$ & $1.12 \mathrm{a}$ & $2.68 \mathrm{bc}$ & $1.13 \mathrm{a}$ & $1.1 \mathrm{a}$ & $3.5 \mathrm{~b}$ & $2.8 \mathrm{a}$ & $2.4 \mathrm{a}$ \\
\hline 50 & $2.58 \mathrm{~b}$ & $1.15 \mathrm{a}$ & $1.12 \mathrm{a}$ & $2.69 \mathrm{bc}$ & $1.10 \mathrm{a}$ & $1.15 \mathrm{a}$ & $2.76 \mathrm{c}$ & $1.15 \mathrm{a}$ & $1.16 \mathrm{a}$ & $3.6 \mathrm{~b}$ & $2.7 \mathrm{a}$ & $2.5 \mathrm{a}$ \\
\hline 100 & $2.63 \mathrm{~b}$ & $1.19 \mathrm{a}$ & $1.18 \mathrm{a}$ & $2.72 \mathrm{bc}$ & $1.16 \mathrm{a}$ & $1.20 \mathrm{a}$ & $2.80 \mathrm{c}$ & $1.21 \mathrm{a}$ & $1.20 \mathrm{a}$ & $3.6 \mathrm{~b}$ & $2.9 \mathrm{a}$ & $2.5 \mathrm{a}$ \\
\hline 150 & $2.77 \mathrm{~b}$ & $1.23 \mathrm{a}$ & $1.27 \mathrm{a}$ & $2.85 \mathrm{bc}$ & $1.20 \mathrm{a}$ & $1.24 \mathrm{a}$ & $2.89 \mathrm{c}$ & $1.28 \mathrm{a}$ & $1.26 \mathrm{a}$ & $3.8 \mathrm{~b}$ & $2.9 \mathrm{a}$ & $2.6 \mathrm{a}$ \\
\hline 200 & $2.81 \mathrm{c}$ & $1.35 \mathrm{a}$ & $1.39 \mathrm{a}$ & $2.88 \mathrm{c}$ & $1.42 \mathrm{a}$ & $1.37 \mathrm{a}$ & $2.93 \mathrm{~cd}$ & $1.54 \mathrm{~b}$ & $1.49 \mathrm{~b}$ & $3.9 \mathrm{~b}$ & $3.0 \mathrm{a}$ & $2.7 \mathrm{a}$ \\
\hline 250 & $2.87 \mathrm{~d}$ & $1.51 \mathrm{~b}$ & $1.52 \mathrm{a}$ & $2.94 \mathrm{de}$ & $1.63 \mathrm{bc}$ & $1.60 \mathrm{a}$ & $2.99 \mathrm{e}$ & $1.69 \mathrm{c}$ & $1.72 \mathrm{c}$ & $4.0 \mathrm{~b}$ & $3.1 \mathrm{a}$ & $2.7 \mathrm{a}$ \\
\hline 300 & $2.93 \mathrm{c}$ & $1.61 \mathrm{a}$ & $1.67 \mathrm{a}$ & $2.97 \mathrm{c}$ & $1.75 \mathrm{ab}$ & $1.72 \mathrm{ab}$ & $3.02 \mathrm{c}$ & $1.82 \mathrm{~b}$ & $1.83 \mathrm{~b}$ & $4.1 \mathrm{~b}$ & $3.2 \mathrm{a}$ & $2.8 \mathrm{a}$ \\
\hline 350 & $2.80 \mathrm{c}$ & $1.72 \mathrm{a}$ & $1.74 \mathrm{a}$ & $3.00 \mathrm{~d}$ & $1.84 \mathrm{a}$ & $1.80 \mathrm{a}$ & $3.08 \mathrm{~d}$ & $1.89 \mathrm{ab}$ & $1.86 \mathrm{ab}$ & $4.3 \mathrm{~b}$ & $3.3 \mathrm{a}$ & $2.9 \mathrm{a}$ \\
\hline 400 & $2.75 \mathrm{~d}$ & $1.63 \mathrm{a}$ & $1.61 \mathrm{a}$ & $2.95 \mathrm{~d}$ & $1.78 \mathrm{~b}$ & $1.73 \mathrm{~b}$ & $3.10 \mathrm{e}$ & $1.95 \mathrm{c}$ & $1.91 \mathrm{c}$ & $4.2 \mathrm{~b}$ & $3.2 \mathrm{a}$ & $2.8 \mathrm{a}$ \\
\hline 450 & $2.72 \mathrm{c}$ & $1.57 \mathrm{a}$ & $1.58 \mathrm{a}$ & $2.86 \mathrm{~d}$ & $1.59 \mathrm{a}$ & $1.64 \mathrm{a}$ & $2.98 \mathrm{~d}$ & $1.99 \mathrm{~b}$ & $2.0 \mathrm{~b}$ & $4.3 \mathrm{~b}$ & $3.2 \mathrm{a}$ & $2.5 \mathrm{a}$ \\
\hline
\end{tabular}

Diferentes letras (horizontalmente dispuestas) dentro de las columnas de cada tratamiento indican diferencias significativas $(P<0.01)$ significativas $(P<0.01)$ significativas $(P<0.01)$ 
La respuesta del suelo al tráfico en las capas más profundas reveló, según lo indicado por el IC, un aumento en el rango de profundidad de 300 a 450 $\mathrm{mm}$ (valores máximos de cuatro años). Todos los valores de IC en el suelo bajo SD después del tráfico, para realizar la labor de siembra, fueron más altos que los valores medidos para $\mathrm{CN}$ y AV. Una razón importante para que el IC sea mayor en el suelo bajo $\mathrm{SD}$ es la baja compresibilidad de este suelo firme, como lo demuestran sus altos valores IC en comparación con los tratamientos de labranza. En el rango de 300 a $450 \mathrm{~mm}$ de profundidad, el promedio de IC en el tratamiento SD, fueron 2.80, 2.94, 3.05 y 4.22 MPa para la primera, segunda, tercera y cuarta estación de crecimiento respectivamente.

Por otra parte, los valores máximos de IC se produjeron a una profundidad cada vez mayor en los últimos años del ensayo. Todos los valores fueron superiores a los citados como críticos para el retraso del crecimiento de raíces (Botta et al., 2004; Joraju- ria et al., 1997). Estos valores de IC indican exceso de compactación en el subsuelo, superando el 1 y 1.2 Mpa límites recomendados por Narro-Farrias (1994) y Terminiello et al. (2000), respectivamente, para evitar el descenso en el rendimiento. Por lo tanto, estos datos apoyan la segunda hipótesis (compactación por los tres métodos de labranza).

\subsection{Materia seca de raíz, materia seca por planta y rendimiento del cultivo de maíz}

La MSR se vio afectada negativamente por la compactación del suelo. A medida que el IC aumentó, MSR disminuyó en el perfil del suelo (Figura 11). Para los tres tratamientos se detectaron diferencias significativas, ocho semanas después de la emergencia de plántulas (en la floración), en el promedio de los valores de MSR. El promedio más alto de MSR fueron para los tratamientos AV y CN (45.9 y $42.2 \mathrm{~g}$ planta $^{-1}$, respectivamente), mientras que el valor más alto en SD fue de $37.0 \mathrm{~g} \mathrm{planta}^{-1}$ (Tabla 6).

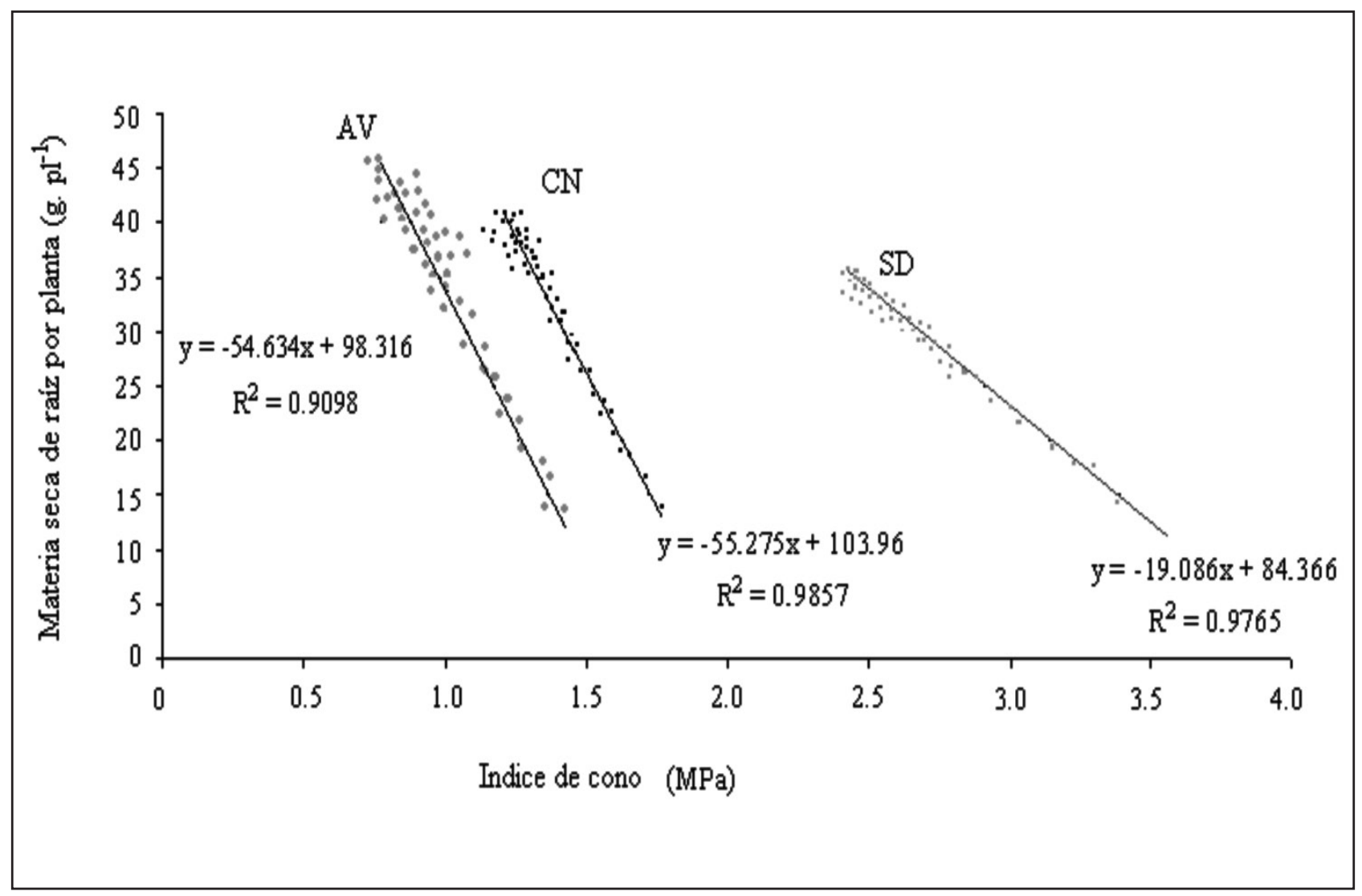

Figura 11. Relación entre la MSR y el IC. 
Tabla 6. MSR y MSP $\left(\mathrm{g}\right.$ planta $\left.^{-1}\right)$

\begin{tabular}{|c|c|c|c|c|c|}
\hline \multicolumn{6}{|c|}{ MSR por planta (g planta ${ }^{-1}$ ) } \\
\hline $\begin{array}{l}\text { Tratamiento } \\
\text { de labranza }\end{array}$ & $\begin{array}{l}1^{\circ} \text { Estación de } \\
\text { crecimiento }\end{array}$ & $\begin{array}{l}2^{\text {do }} \text { Estación } \\
\text { de crecimiento }\end{array}$ & $\begin{array}{l}3^{\text {tro }} \text { Estación } \\
\text { de crecimiento }\end{array}$ & $\begin{array}{c}4{ }^{\text {ta }} \text { Estación } \\
\text { de crecimiento }\end{array}$ & Promedio \\
\hline SD & $37.0 \mathrm{a}$ & $37.3 \mathrm{a}$ & $37.1 \mathrm{a}$ & $36.9 \mathrm{a}$ & $37.0 \mathrm{a}$ \\
\hline $\mathrm{CN}$ & $43.0 \mathrm{~b}$ & $42.3 \mathrm{~b}$ & $41.6 \mathrm{~b}$ & $42.1 \mathrm{~b}$ & $42.2 \mathrm{~b}$ \\
\hline AV & $47.2 \mathrm{c}$ & $46.1 \mathrm{c}$ & $45.0 \mathrm{c}$ & $45.3 \mathrm{c}$ & $45.9 \mathrm{c}$ \\
\hline \multicolumn{6}{|c|}{$\operatorname{MSP}\left(\right.$ g planta $\left.^{-1}\right)$} \\
\hline SD & $142.4 \mathrm{a}$ & $143.2 \mathrm{a}$ & $141.0 \mathrm{a}$ & $142.5 \mathrm{a}$ & $142.3 \mathrm{a}$ \\
\hline $\mathrm{CN}$ & $152.0 \mathrm{~b}$ & $153.6 \mathrm{~b}$ & $150.2 \mathrm{~b}$ & $151.9 \mathrm{~b}$ & $151.9 \mathrm{~b}$ \\
\hline AV & $158.4 \mathrm{c}$ & $160.0 \mathrm{c}$ & $159.0 \mathrm{c}$ & $160.2 \mathrm{c}$ & $159.4 \mathrm{c}$ \\
\hline
\end{tabular}

Diferentes letras (entre tratamiento de labranza) indican diferencias significativas $(P<0.01)$

La Figura 12 muestra que, después del tráfico de siembra, la relación entre la MSR y el RM fue muy positiva.

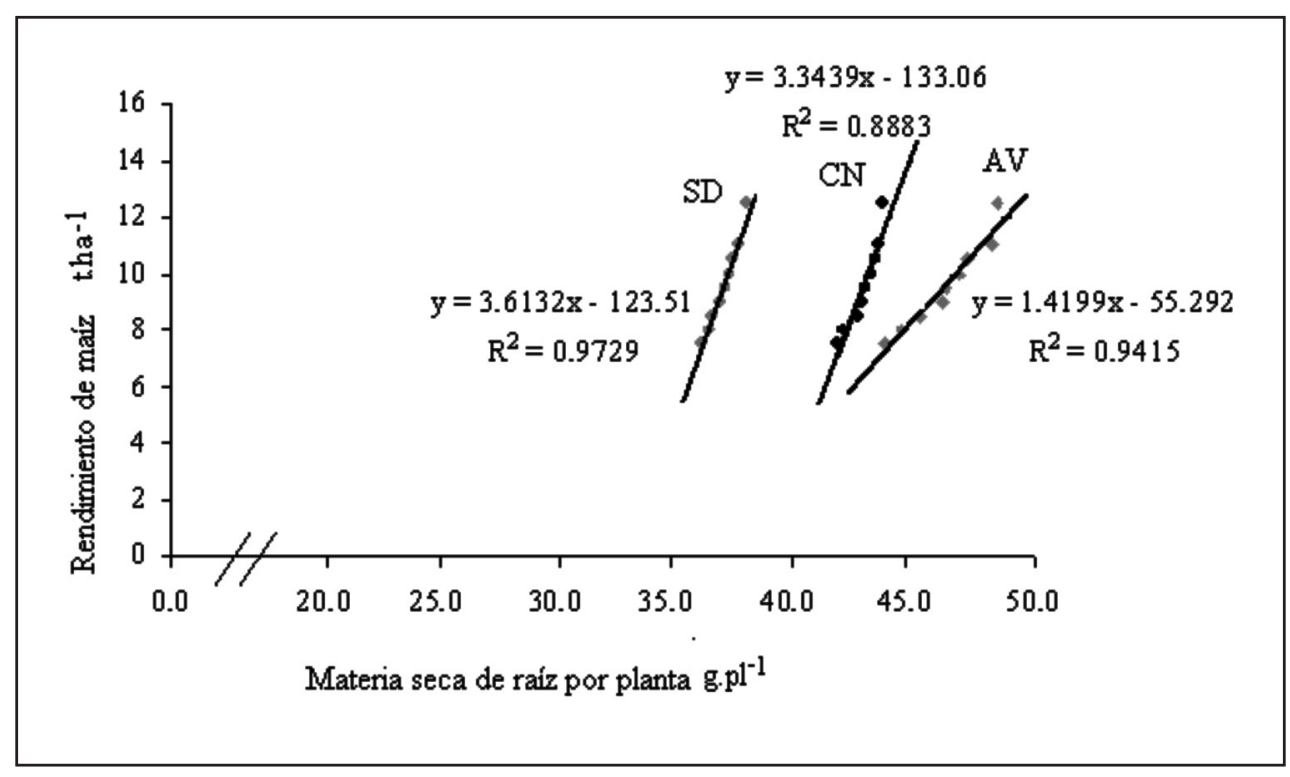

Figura 12. Relación entre el rendimiento del maíz y la MSR.

En todos los tratamientos, la MSR fue directamente proporcional a los RM, con un coeficiente de regresión alto. Por otra parte, los RM fueron mayores en $\mathrm{CN}$ y $\mathrm{AV}$.

Los valores más bajos de compactación del suelo y de esfuerzo cortante sobre el suelo arado (en este caso $10.01 \mathrm{kN}$ ) se asociaron con una mayor producción, seguido de CN (16.0 kN) y SD $(22 \mathrm{kN})$. Estos resultados confirman las dos hipótesis.
Para las cuatro estaciones de crecimiento, la MSP fue estadísticamente diferente para cada tratamiento. El promedio de la MSP fue $159.4 \mathrm{~g}$ planta $^{-1}$ en $\mathrm{AV}$, seguido por $151.9 \mathrm{~g}$ planta $^{-1}$ en CN y 142.3 g planta $^{-1}$ en SD.

Los mejores resultados para la emergencia de las plántulas fueron producidas en el suelo bajo SD (Tabla 4). Sin embargo, los RM fueron mayores en CN y AV (Tabla 7), con un decremento significativo de 12.3 y $17.5 \%$, de SD respecto a CN y AV. 
Tabla 7. Rendimiento del maíz ( $\left.\mathrm{t} \mathrm{ha}^{-1}\right)$

\begin{tabular}{|cccc|}
\hline $\begin{array}{c}\text { Cosecha / } \\
\text { Tratamientos }\end{array}$ & SD & CN & AV \\
\hline 2005 & $9.9 \mathrm{~b}$ & $10.8 \mathrm{a}$ & $11.1 \mathrm{a}$ \\
2006 & $10.1 \mathrm{~b}$ & $11.6 \mathrm{a}$ & $11.9 \mathrm{a}$ \\
& & & \\
2007 & $10.0 \mathrm{~b}$ & $11.2 \mathrm{a}$ & $12.5 \mathrm{a}$ \\
& & & \\
2008 & $9.7 \mathrm{~b}$ & $11.5 \mathrm{a}$ & $12.6 \mathrm{a}$ \\
& & & \\
& $9.9 \mathrm{~b}$ & $11.3 \mathrm{a}$ & $12.0 \mathrm{a}$ \\
\hline
\end{tabular}

Diferentes letras (entre tratamiento de labranza) indican diferencias significativas $(P<0.01)$

A pesar de que la compactación del suelo es mucho mayor en los primeros $200 \mathrm{~mm}$ en SD que en $\mathrm{CN}$ y AV, las diferencias en los RM fueron pequeñas pero estadísticamente significativas El factor más importante puede haber sido que los patrones de las precipitaciones fueron particularmente favorables para el crecimiento del maíz. Estas lluvias favorables deben haber "anulado", por así decirlo, el mayor nivel de compactación en el tratamiento bajo SD.

\section{Conclusiones}

A pesar del menor número de pasadas del tratamiento bajo SD, los rendimientos de maíz fueron, de año en año, menores a los otros dos tratamientos.

El alto peso $(187 \mathrm{kN})$ del equipo utilizado par el tratamiento de SD produjo compactación del subsuelo, que llevó a cambios en el sistema de la

\section{Referencias}

AAPRESID. (2013). Manual de buenas prácticas agricolas e indicadores de gestión. Rosario: Asociación Argentina de Productores en siembra directa. raíz del maíz, y estos cambios no significan beneficios para su rendimiento.

Los valores medidos de IC demostraron que la compactación del subsuelo persistió durante más de 3 años.

El rendimiento del maíz tuvo relación directa con la materia seca de raíz, la cual fue afectada por la compactación del suelo producida por el tráfico de los equipos utilizados.

A pesar de las diferencias en la cobertura de rastrojo se encontró que, entre los tratamientos de labranza, la emergencia de las plántulas de maíz no se vio afectada.

Las sembradoras de bajo peso utilizadas en los tratamientos $\mathrm{AV}$ y $\mathrm{CN}$ produjeron compactación subsuperficial del suelo.
Al-Adawi, S., \& Reeder, R. (1996). Compaction and subsoiling effects on corn and soybean yields and soil physical properties. Transactions of the ASAE, 39(5), 1641-1649. 
ASAE Standard. (1993). Terminology and definitions for soils tillage and soil tool relationships Engineering Practice, EP291. 2. American Society of Agricultural Engineering.

ASAE Standards. (1992). Soil cone penetrometer $S$ 313.2. American Society of Agricultural Engineering.

Blevins, R., Thomas, G., \& Cornelius, P. (1977). Influence of no tillage and $\mathrm{N}$ fertilization on certain soil properties after 5 years of continuous corn. Agronomy Journal, 69, 383-386.

Botta, G. (2000). Subsoil compaction distribution induced by agricultural traffic. 230. (L. University, Ed.) Argentina.

Botta, G., Jorajuría, D., \& Draghi, M. (2002). In fluence of the axle load, tyre size and configuration on the compaction of a freshly tilled clayey soil. Journal of Terramechanics, 39(1), 47-54.

Botta, G., Jorajuria, D., \& Rosatto, H. (2006). Light tractor frequency on soil compaction in the rolling Pampa region of Argentina. Soil \& Tillage Research, 86, 9-14.

Botta, G., Jorajuria, D., Balbuena, R., \& Rosatto, H. (2004). Mechanical and cropping behaviour of direct drilled soil under different traffic intensities: Effect an soybean (Glycine max L.) yields. Soil \& Tillage Research, 78, 53-58.

Botta, G., Jorajuria, D., Balbuena, R., Ressia, M., Ferrero, C., Rosatto, H., \& Tourn, M. (2006). Deep tillage and traffics on subsoil compaction and sunflower (Helianthus annus L.). Soil \& Tillage Research, 91, 164-172.

Botta, G., Tolón-Becerra, A., Bellora, F., \& Tourn, F. (2009). Seedbed compaction produced by traffic on four tillage regimes in the rolling Pampas of Argentina. Soil \& Tillage Research, 105, 128-134.

Botta, G., Tolón-Becerra, A., Lastra-Bravo, X., \& Tourn, M. (2010). Tillage and traffic effects (planters and tractors) on soil compaction and soybean (Glycine max L.) yields in Argentinean pampas. Soil \& Tillage Research, 110, 167-174.

Botta, G., Tolón-Becerra, A., Lastra-Bravo, X., Tourn, M., Balbuena, R., \& Rivero, D. (2013). Continuous application of direct sowing: Traffic effect on subsoil compaction and maize (Zea mays L.) yields in Argentinean Pampas. Soil \& Tillage Research, 134, 111-120.

Edwards, J., Wood, W., \& Ruf, M. (1992). Tillage and crop rotation effects on fertility status of a Hapludult soil. Soil Science Society American Journal, 56, 1577-1582.

Flowers, M., \& Lal, R. (1998 ). Axle load and tillage effects on soil physical properties and soybean grain yield on a mollic achraqualf in northwest Ohio. Soil \& Tillage Research, $48,21-35$.

Fonterosa, R. (2012). Efectos de diferentes métodos de labranza sobre la compactación del suelo y el rendimiento del maíz (Zea Mays L.) en el este de la región pampeana. 32 .

Håkansson, I., \& Reeder, R. (1994). Subsoil compaction by vehicles with high axle load-extent, persistence and crop response. Soil and Tillage Research, 29, 277-304.

Hill, R. (1990). Long term conventional and no tillage effects on selected soil physical properties. Soil Science Society American Journal, 54, 161-166.

Horn, R. (1998). Effect of agregation and tillage systems on soil deformation by mechanical strees and strain. Advances in Sugar Beet research, 1, 7-20.

Jorajuria, D., Draghi, L., \& Aragon, A. (1997). The effect of vehicle weight on the distribution of compaction with depth and the yield of Lolium (Trifolium grassland). Soil \& Tillage Research, $41,1-12$.

Narro-Farrias, E. (1994). Física de suelos, con enfoque agrícola. Mexico: Editorial Trillas. 
Oskui, K., \& Voorhees, B. (1990). Prediction of planting timeliness penalties for corn and soybean in northern corn belt. Agron. Abstr. (pág. 153). Madison, WI, USA.

Söhne, W. (1958). Fundamentals of pressure distribution and soil compaction under tractors tyres. Agricultural Engineering, 39, 276-281.

Soil Conservation Service. (1994). Keys to Soil Taxonomy (6th ed.). (S. S. Staff, Ed.) Washington DC, USA: USDA.

Taylor, H., Roberson, G., \& Parker, J. (1966). Soil strength root penetration relations for medium coarse-textured soil materials. Soil Science, 102, 18-22.

Terminiello, A., Claverie, J., Casado, J., \& Balbuena, R. (2000). Cone index evolution through the growth season of cabbage crop (Brassica oleracea L.). Proc 4th CADIR (Argentine Congress on Agricultural Engineering), 1, págs. 68-73.

Tourn, M., Soza, E., Botta, G., \& Mete, A. (2003). Direct maize seeding. Effects of residue clearance on implant efficiency. Spanish Journal of Agricultural Research, 1, 99-103.

USDA (United States Department of Agriculture). (January de 2013). Oilseeds 13-01. Circular Series.

Voorhees, B., \& Lindstrom, J. (1983). Soil compaction constraints on conservation tillage in the northern corn belt. Journal of Soil Water conservation, 38, 307-311. 